\title{
Diagnostic Challenges Revealed from a Neuropsychiatry Movement Disorders Clinic
}

\author{
Heather Rigby, Angela Roberts-South, Hrishikesh Kumar, \\ Leonardo Cortese, Mandar Jog
}

\begin{abstract}
Background: Abnormal movements are frequently associated with psychiatric disorders. Optimized management and diagnosis of these movements depends on correct labeling. However, there is evidence of reduced accuracy in the labeling of these movements, which could result in sub-optimal care. Objective: To determine the consensus inter-rater reliability between a movement disorders neurologist and physicians referring from the community for phenomenology and diagnoses of individuals with co-existing psychiatric conditions and movement disorders. Method: Charts of all consecutive patients seen in a combined Movement Disorders and Neuropsychiatry Clinic between 2001-2009 were reviewed retrospectively. Consensus estimates and kappa values for inter-rater reliability were determined for phenomenology and diagnostic terms for the respective referring source and movement disorders neurologist for each patient. Results: A total of 106 charts were reviewed (62 men and 44 women). Agreement for phenomenology terms ranged from $0 \%$ (psychogenic) to $73 \%$ (tremor). Only 3 terms had kappa values that met or exceeded criteria for moderate inter-rater reliability. Agreement for diagnosis terms was highest for tardive dyskinesia (83\%), drug induced tremor (33\%), and drug induced parkinsonism (20\%). In 18 of the 22 charts (82\%), a diagnosis was made of drug induced movement disorder (DIMD) by the referring physician. In contrast, a diagnosis of DIMD was made in only 54 of 106 charts (51\%) after the patients were assessed in the clinic. Conclusions: A movement disorders specialist frequently disagreed with referring physicians' identification of patient phenomenology and diagnosis. This suggests that clinicians would benefit from educational resources to assist in characterizing abnormal movements.
\end{abstract}

RÉSUMÉ: Défis diagnostiques identifiés dans une clinique de troubles du mouvement en neuropsychiatrie. Contexte : Des mouvements anormaux sont souvent associés à des troubles psychiatriques. L'optimisation du traitement et du diagnostic de ces mouvements dépend de l'exactitude de leur identification. Cependant, certaines données indiquent que l'exactitude de l'identification de ces mouvements a diminué, ce qui peut entraîner un traitement sous-optimal. Objectif : Le but de l'étude était de déterminer la fiabilité du consensus interobservateurs d'un neurologue spécialiste des désordres du mouvement et de médecins qui réfèrent les patients, concernant les termes utilisés pour identifier la phénoménologie et le diagnostic respectivement par le médecin référant et le neurologue de la clinique du mouvement pour chaque patient. Résultats : Nous avons révisé les dossiers de 106 patients, 62 hommes et 44 femmes. Les termes utilisés pour identifier la phénoménologie concordaient de 0\% (psychogénique) à 73\% (tremblement). Seulement 3 termes avaient des valeurs kappa qui rencontraient ou excédaient les critères de fiabilité inter évaluateurs modérée. La concordance pour les termes du diagnostic était plus élevée pour les dyskinésies tardives (83\%), le tremblement induit par la médication (33\%) et le parkinsonisme induit par la médication (20\%). Chez 18 des 22 patients $(82 \%)$, un diagnostic de trouble du mouvement induit par un médicament (TMIM) a été posé par le médecin référant. Par contre, un diagnostic de TMIM a été posé chez seulement 54 des 106 patients (51\%) suite à leur évaluation à la clinique. Conclusions : L'opinion d'un spécialiste des troubles du mouvement était souvent en désaccord avec celle du médecin référant quant à l'identification de la phénoménologie et du diagnostic chez le patient. Il semble donc que les cliniciens bénéficieraient de ressources éducatives pour les aider à caractériser les mouvements anormaux.

Can J Neurol Sci. 2012; 39: 782-788

Abnormal involuntary movements are frequently associated with psychiatric disorders. They can be the result of medication exposure or seen as part of the symptom complex of a primary psychiatric illness. Conversely, primary movement disorders may have a psychiatric presentation (as in Huntington's disease). Psychogenic movement disorders cross the boundaries of these two disciplines. The shared neurochemical basis of psychiatric illness and movement disorders may partially account for their frequent association.

Accurate diagnosis of movement disorders in individuals with concomitant psychiatric diagnoses can be challenging.
Hansen, et al reported the under-recognition of tardive dyskinesia (TD) and drug-induced parkinsonism (DIP) by resident physicians ${ }^{1}$. Of 101 patients in the study, 28\% had TD

From Dalhousie University (HR), Halifax, Nova Scotia; University of Western Ontario (ARS, HK, LC, MJ); London Health Sciences Centre (ARS, HK, LC, MJ), London, Ontario, Canada.

Received November 29, 2011. Final Revisions Submitted June 29, 2012. Correspondence to: Angela Roberts-South, c/o Mandar Jog. 334 Windermere Blvd. A10-026, London, Ontario, N6A 5A5, Canada.Email: Asouth4@uwo.ca. 
and $26 \%$ had DIP of which the residents only correctly identified TD in $12 \%$ and DIP in $11 \%$ of the sample. Additionally, Lauterbach, et al reported that of 49 hospitalized patients with TD seen for movement disorder consultation, $23.9 \%$ had movement disorders other than $\mathrm{TD}^{2}$. Collectively these studies demonstrate that there are issues with consistency in diagnosing movement disorders in a psychiatric population.

The ability to accurately identify abnormal movements according to accepted phenomenology is critical for making a diagnosis in movement disorders. Furthermore, failure to accurately identify phenomenology may compromise patient care through incorrect or delayed determination of the movement disorder diagnosis resulting in compromised management. For example, research has demonstrated that in DIP the symptoms usually regress with discontinuation of the offending medication, however, in $15 \%$ of cases symptoms persisted after drug withdrawal ${ }^{3,4}$. Further, the detection of parkinsonism in patients with a co-existing hyperkinetic movement disorder is often managed differently from an otherwise isolated hyperkinetic condition. Presynaptic dopamine depleting medications such as tetrabenazine are often used in the treatment of hyperkinetic movement disorders but can worsen underlying parkinsonism. These studies underscore the importance of accurate use of phenomenology for determining diagnosis and subsequent management.

\section{OBJective}

To further investigate the phenomenon of accurate use of phenomenology and diagnostic labels in movement disorders, the objective of the current study was to investigate the interrater reliability of phenomenology used to describe abnormal movements and the diagnostic labels assigned to these movements between community practice physicians and a movement disorders neurologist. This work aims to extend previous research in the area by: 1) addressing both phenomenology and diagnostic labels, 2) sampling patients from a variety of psychiatric and movement disorders without limiting selection to those with drug induced conditions, and 3) sampling patients from a community dwelling vs. hospitalized population.

\section{Method}

This is a retrospective chart review study. Research was approved by and conducted in accordance with the guidelines of the Human Subject Research Ethics Board (HSREB) at the University of Western Ontario.

\section{Chart Review}

The charts of all consecutive patients, with an underlying psychiatric disorder, seen in a joint Neuropsychiatry and Movement Disorders (MD) clinic in the MD clinic between 2001 and 2009 were reviewed for the presence of referral source documentation (letter, specific referral form, medical visit note) and the initial movement disorders neurologist (MDN) assessment documentation. Only charts containing both referral source and MDN documentation were included in the study. A movement disorder neurologist (MJ) and a neuropsychiatrist (LC) conducted the joint clinic. The referral source documentation was used to extract data for phenomenology and diagnostic labels assigned by community physicians. The movement disorders consult note was used to extract data for phenomenology and diagnostic labels assigned by a movement disorders neurologist.

Data was collected from the charts by study authors (HR) and (HK) and was fully de-identified to protect confidentiality of the participants. Neither of the researchers was involved in the initial care or documentation of care from which data was collected. Data collected from each medical record included: all referral source and MD clinic phenomenology and diagnostic terms, psychiatric diagnosis, current and previous medications, duration of movement disorder, duration of psychiatric disorder, referral source, age, other relevant medical history (head injury, etc.), clinic follow-up recommendations, and relevant life-style factors (smoking, alcohol use, etc.).

The primary variables of interest were MD clinic phenomenology and diagnostic labels versus referral source phenomenology and diagnostic labels appearing in the medical documentation. Once all phenomenology and diagnostic terms were extracted from the chart, they were collated into a single list independent of their data sources. Two neurologists (one movement disorders specialist and one movement disorders fellow) assigned each of the terms to either the phenomenology or diagnosis category. One hundred percent consensus on whether terms fit into either the phenomenology or diagnosis category was required prior to completing data analysis. Phenomenology and diagnostic terms that did not reach full consensus on initial category assignment were discussed between raters until full consensus was reached.

Referral source documentation that listed a combination of at least two of the phenomenology terms "tremor" + "gait difficulty/shuffling" + "stiffness/rigidity/slowness" were considered to be in agreement with the term "parkinsonism" applied by MD clinic even if the referral source did not specifically use the term "parkinsonism". Otherwise a precise match of phenomenology terms was required for agreement (e.g. abnormal movement from referral source was not considered in agreement with dyskinesia in the MD clinic note).

\section{Statistical Analysis}

To describe the typical population seen in the clinic we selected a descriptive statistics method with $95 \%$ confidence interval. Variables of interest included gender, age, psychiatric diagnosis, duration psychiatric symptoms, duration abnormal movements, medications, and referral source.

To answer the question of whether movement disorders neurologists and physicians referring patients from the community use the same construct and diagnostic rubric to describe abnormal movements and to assign an appropriate diagnostic label, we used consensus estimates and kappa values for inter-rater reliability. The phenomenology and diagnostic Kappa values were calculated by comparing the respective labels provided by the referring physician and the movement disorders specialist for each patient.

For consensus estimates, simple percent agreement statistics were calculated for each phenomenology and diagnostic term appearing in the medical notes in addition to a total simple percent agreement for all phenomenology and diagnostic terms. Raters were defined as MD clinic versus referral source. 


\section{Table 1: Distribution of psychiatric diagnosis by percentage of total diagnoses}

\begin{tabular}{ll}
\hline $\begin{array}{l}\text { Percentage of Total } \\
\text { Diagnoses }\end{array}$ & Diagnosis $\mathrm{I}$ \\
\hline $33 \%$ & Affective Disorders \\
\hline $23 \%$ & Schizophrenia \\
\hline $14 \%$ & Bipolar Disorder \\
\hline $10 \%$ & Schizoaffective Disorder \\
\hline $3 \%$ (each) & Psychosis (not otherwise specified), Uncertain \\
\hline $2 \%$ (each) & Post-traumatic Stress Disorder, Obsessive \\
& Compulsive Disorder, Panic Disorder, \\
& Personality Disorder, Conversion, \\
& Autism/Pervasive Developmental Disorder \\
\hline $1 \%$ (each) & Multiple Personality, Attention Deficit \\
& Hyperactivity Disorder \\
\hline
\end{tabular}

$\dagger$ Psychiatric diagnoses made by a psychiatrist (LC) based on the Diagnostic and Statistical Manual of Mental Disorders, 4th edition (DSM-IV); American Psychiatric Association. Diagnostic and Statistical Manual of Mental Disorders, Revised 4th edition. Washington, DC: Author; 2000.

Consensus inter-rater reliability for all calculations was based on agreement of the referral source phenomenology/diagnosis to the MD clinic phenomenology/diagnosis. A reliability of $70 \%$ or greater was considered acceptable ${ }^{5}$.

Kappa coefficients for inter-rater reliability (MD clinic vs. referral source) were calculated for each phenomenology and diagnostic label. Interpretation of agreement based on Kappa values follows: < 0 less than chance, $.01-.20$ slight, .21-.40 fair, $.41-.60$ moderate, $.61-.80$ substantial, and $.81-.99$ almost perfect.

\section{RESULTS}

\section{Charts Reviewed}

A total of 112 charts were reviewed. Six were excluded because the necessary medical documentation was not present for accurate data collection. A total of 106 charts were analyzed for phenomenology and diagnosis. Referral sources included: psychiatrists $(75.55 \%, \mathrm{n}=80)$, family physicians $(18.87 \%, \mathrm{n}=$ $20)$, and neurologists $(5.64 \%, \mathrm{n}=6)$.

\section{Demographics}

Data from charts of $62(58 \%)$ men and $44(42 \%)$ women were analyzed. All individuals were community dwelling. Ages ranged from 11 to 86 years $(\mathrm{M}=54.11, \mathrm{SD}=17.7,95 \% \mathrm{CI}$ $[50.74,57.48])$. The distribution across age groups was $4 \%(n=4)$ under the age of 20 years, $18 \%(n=19)$ between ages of 21 and 40 years, $32 \%(\mathrm{n}=34)$ between ages of $41-60$ years, and $46 \%$ $(n=49)$ age 61 years or greater.
Table 2: Distribution of psychiatric medications extracted from charts reviewed

\begin{tabular}{ll}
\hline Percentage & Medication \\
\hline $26 \%$ & Atypical Antipsychotics \\
\hline $16 \%$ & Anticonvulsants \\
\hline $16 \%$ & SSRI/SNRI/NaSSA/Bupropion/Trazadone \\
\hline $14 \%$ & Benzodiazapines \\
\hline $6 \%$ & Dopaminergic \\
\hline $5 \%$ & Anticholinergics \\
\hline $4 \%$ & Hypnotics \\
\hline $4 \%$ & Lithium \\
\hline $4 \%$ & Typical Antipsychotics \\
\hline $3 \%$ & Tricyclic Antidepressants \\
\hline $1 \%$ & Cholinergic \\
\hline
\end{tabular}

A total of 13 different psychiatric diagnoses were reported. The breakdown of cases by psychiatric diagnosis appears in Table 1. Twenty-four $(22.6 \%)$ charts had more than one psychiatric diagnosis listed. Chart review indicated that $26 \%$ of medications extracted from the chart review were atypical antipsychotics. A full breakdown of all medications appears in Table 2.

The mean duration of psychiatric diagnosis was determined from 60 charts $(56.6 \%)$. The remaining charts did not have a clearly identifiable duration with $44(41.5 \%)$ charts reporting duration of "many years", $1 \quad(<1 \%)$ chart reporting an "unknown" duration and $1(<1 \%)$ chart reporting "a few years". From the 60 charts used in the calculation, the duration of psychiatric diagnosis ranged from 0.5 to 59 years $(\mathrm{M}=14.0, \mathrm{SD}$ $=12.89,95 \% \mathrm{CI}[10.74,17.26)$. However, the duration reported here may be skewed lower than the true duration based on a substantial number of missing data points labeled "many years" suggesting a longer history of illness.

\section{Duration of Abnormal Movements}

The duration of abnormal movements was determined from 98 of $106(92.5 \%)$ charts. Of the eight charts without a discernable duration, two had no duration reported and six had a qualifier of "many years" or "decades" without the number of years specified. The duration of the movement disorder ranged from 0.3 to 25 years with a mean of 5.2 years $(\mathrm{SD}=1.56,95 \%$ CI $[4.89,5.51])$. This suggests a five year delay between onset of movement and referral to a movement disorders specialist.

\section{Phenomenology Consensus}

All 106 charts had at least one phenomenology term listed by the referral source and by the MD clinic documentation. To 


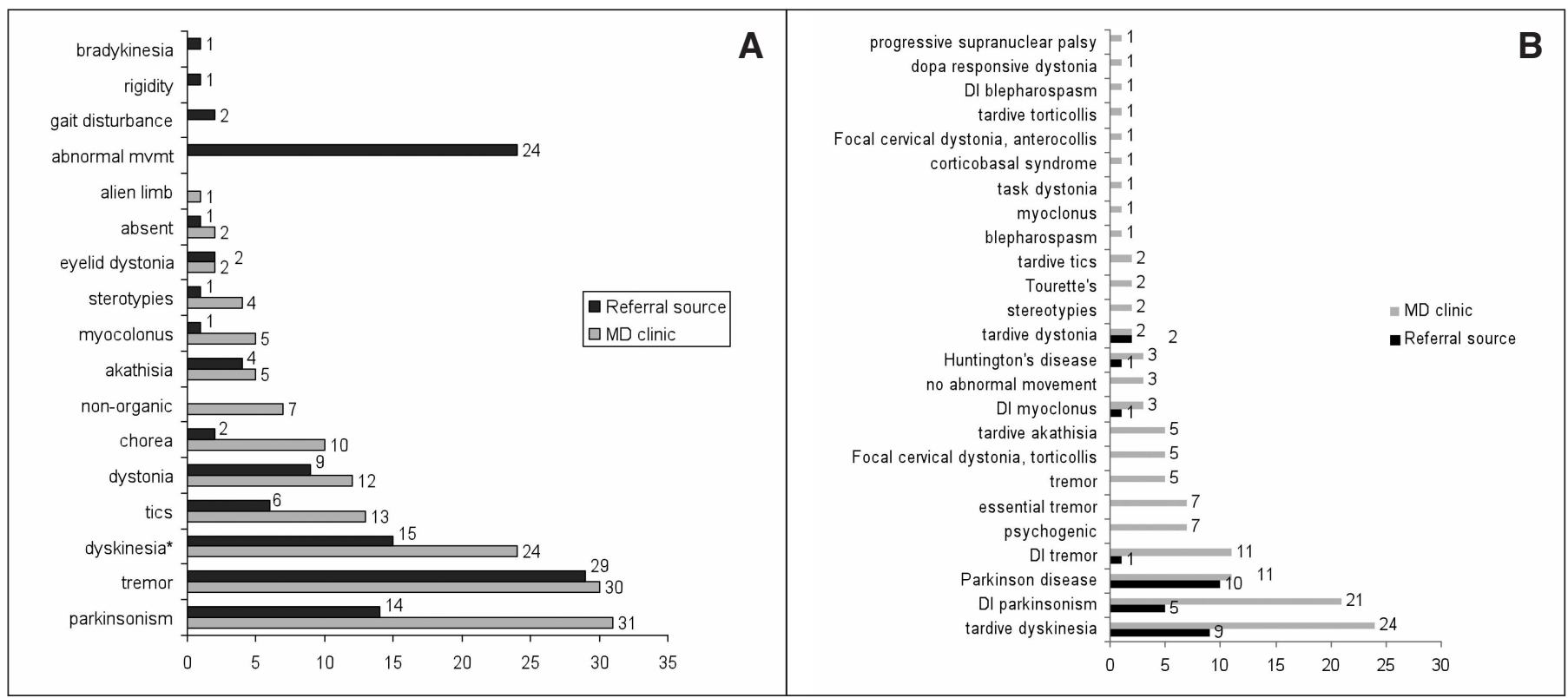

Figure 1: Distribution (raw number) of Phenomenology (A). * Note. "Dyskinesia" is not a generally accepted phenomenology term for describing movements, but is a more global term used for a family of phenomenological terms. However, in the charts reviewed it was used by both the referring and the movement disorder physician as a phenomenology term to describe complex hyperkinetic movements involving single or multiple body parts which were not classifiable using other phenomenology terms. To preserve the data, the authors have used it as a phenomenological term because it was the actual term used in the clinical record.

determine consensus between the referral source phenomenology and the MD clinic phenomenology, 162 occurrences of terms were entered into analysis. Multiple charts had more than one phenomenology listed $(\mathrm{n}=45,42 \%)$. The distribution of phenomenology by the referral source is reported in Figure $1 \mathrm{~A}$.

Concordant agreement by phenomenology was: $73 \%$ for tremor, $54 \%$ for dyskinesia, $50 \%$ for blepharospasm, $31 \%$ for tics, $29 \%$ for parkinsonism, $25 \%$ for dystonia, $20 \%$ for chorea, and $0 \%$ for akathisia, myoclonus, alien limb, stereotypies, nonorganic movements (psychogenic), and absence of abnormal movement. Corresponding kappa statistics for inter-rater reliability ranged from .724 to 0 (Figure 2A). Only three phenomenology terms tremor (.724), eyelid dystonia (.652), and dyskinesia (.565) had kappa values that met or exceeded criteria for moderate inter-rater reliability. The mean overall inter-rater reliability kappa value for phenomenology was $.273(\mathrm{SD}=.276$, $95 \%$ CI $[.12, .42])$.

\section{Diagnostic Consensus}

Of the 106 charts, 28/106 (26\%) of referral source documentation provided a diagnostic label to the case. Of the MD clinic notes 93/106 (88\%) provided at least one diagnostic label to the chart. This discrepancy may suggest that community referral sources were not comfortable providing diagnostic labels for movement disorders. The distribution of diagnosis reported by the MD clinic when the referral source did not provide a diagnostic label appears in Figure 3. Thirty-five charts (33\%) had multiple diagnostic labels reported in the referral source and/or the MD clinic documentation with a total of 117 occurrences of diagnostic terms across all charts. The

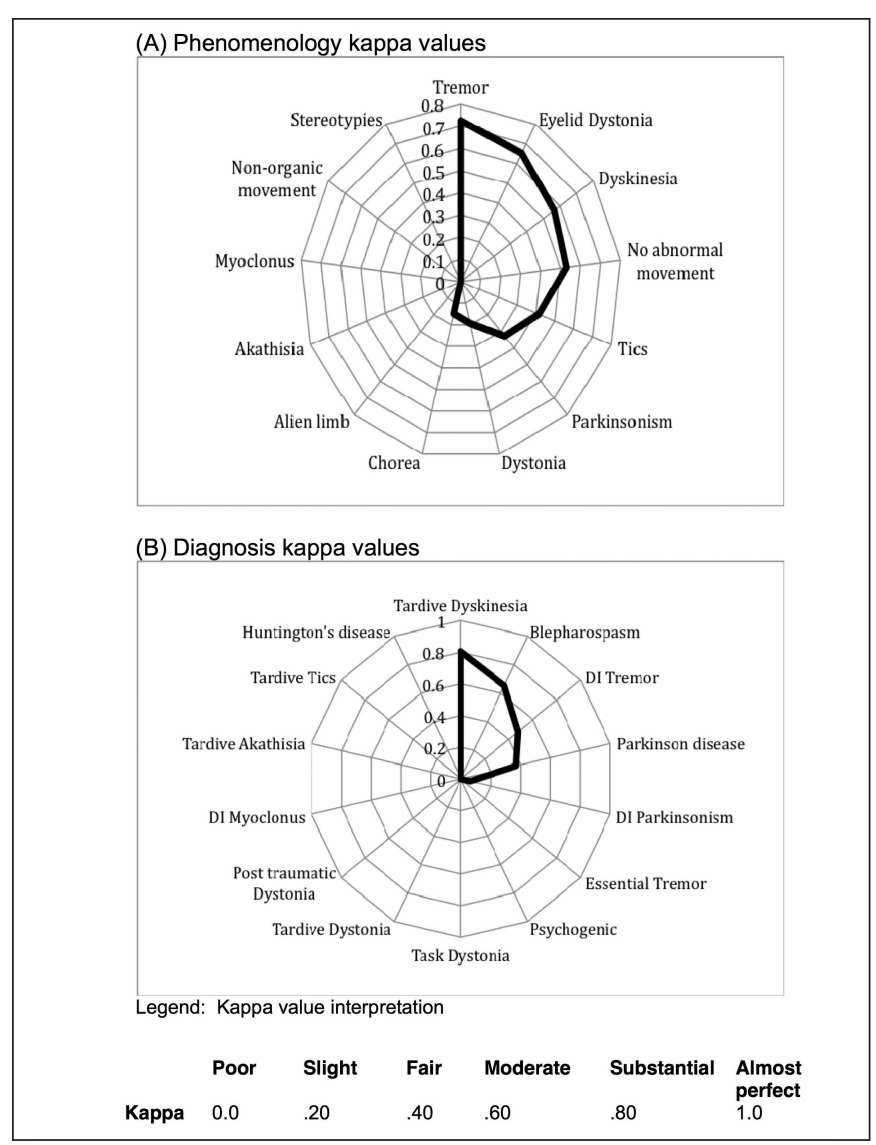

Figure 2: Kappa Statistic Radar Plots of Inter-rater Reliability for Phenomenology (A) and for Diagnosis (B). Distribution (raw number) of Diagnostic Terms by Movement Disorders Clinic vs. Referral Source 


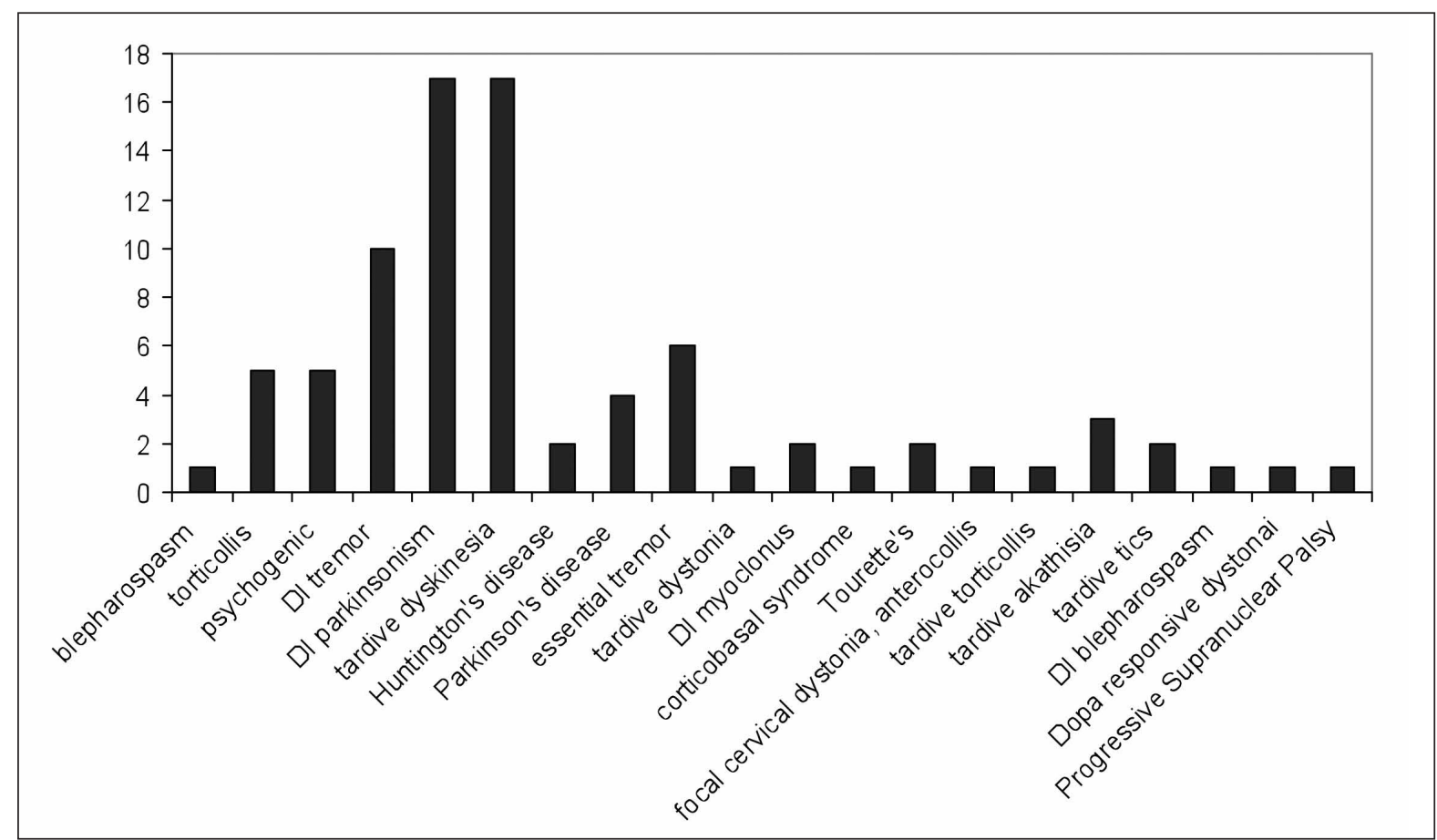

Figure 3: Diagnosis made by movement disorders specialist when no diagnosis provided by referral source

distribution of diagnoses from the referral source and the MD clinic documentation are shown in Figure 1B.

From the 28 charts listing diagnostic terms for both the referral source and the MD clinic, six (five with idiopathic Parkinson's disease and one with Huntington's disease) were excluded from the consensus study because the movement disorder was diagnosed prior to referral to the MD clinic and were felt to overestimate the consensus for Parkinson's disease and Huntington's disease. Consensus inter-rater reliability statistics were calculated on $22(21 \%)$ of the charts reviewed reporting 33 diagnostic terms (28.2\% of total diagnostic terms). Consensus agreement for each diagnosis was: $83 \%$ tardive dyskinesia, 33\% drug induced tremor, 20\% drug induced parkinsonism, and $0 \%$ for psychogenic movement disorder, task specific dystonia, idiopathic Parkinson's disease, essential tremor, tardive dystonia, tardive akathisia, tardive tics, drug induced myoclonus, and absence of abnormal movement. Corresponding kappa statistics ranged from .805 to 0 (Figure 2B). Only two diagnosis terms, tardive dyskinesia (.805) and blepharospasm NOS (.724), had kappa values that met or exceeded criteria for moderate inter-rater reliability.

A diagnosis of drug-induced movement disorder (DIMD) was made in 54/93 (58\%) charts by the movement disorders neurologist. In contrast, 18/22 (82\%) diagnoses provided by referral sources were DIMDs. Multiple MD clinic notes reported the co-occurrence of drug induced and non-drug induced movement disorders within the same patient $(n=9,8 \%)$. This suggests a bias in assignment of drug induced aetiologies by community referral sources. As well this data reinforces the cooccurrence of drug induced and non drug induced movement disorders in persons with psychiatric illness. The mean overall inter-rater reliability kappa value for diagnosis was .169 (SD = $.283,95 \%$ CI $[.02, .32])$.

\section{Discussion}

The present study provides evidence that in a sample of patients with psychiatric illness and concomitant movement disorders there is a lack of consistency in the phenomenology terms used to describe abnormal movements between community physicians and a movement disorders clinic. Additionally, this research provides evidence of poor consistency in the diagnosing of movement disorders between community physicians compared to a movement disorders clinic. A number of factors may contribute to difficulty identifying correct phenomenology. Clinical findings may be missed because of fluctuating clinical appearance of abnormal movements, lack of proficiency in movement disorder physical examination, and uncertainty regarding mild clinical findings ${ }^{6,7}$.

A significant number of patients $(24 / 106 ; 23 \%)$ were not described using specific phenomenological terms in the referral letters. Rather, the referring physicians often used the nonspecific term "abnormal movement". However, all movements could be classified into a definitive phenomenological category by MD clinic neurologist. The exact source of this discrepancy is unclear from the data. This may reflect a movement disorders phenomenology knowledge "gap" between community physicians and MD neurologists. Alternatively, this finding may be biased by the data source, referring physician notes. It is possible that the time demands within daily clinical practice and additionally the fact that community physicians were referring to a specialty clinic resulted in referral source documentation that did not fully reflect their analysis of the patient. 
Only three phenomenology and two diagnostic labels reported in the charts had kappa values that met criteria for moderate agreement between clinicians. Additionally only one phenomenology (tremor) and one diagnosis (tardive dyskinesia) met criteria for substantial inter-rater agreement. While we studied a much larger sample of movement disorders in our agreement study as well as studying both phenomenology and diagnosis labels, our findings are in keeping with Lauterbach, et al who reported agreement concerns with the diagnosis of movement disorders between physicians in a state psychiatric hospital and a consulting movement disorders service ${ }^{2}$.

Many movement disorders are associated with significant morbidity and mortality. Tardive dyskinesia, which affected $23 \%$ of the patients in our study, confers a significant increased mortality ${ }^{8}$. Two cases of Huntington's disease were misdiagnosed as having tardive dyskinesia. These errors in diagnosis can result in substantial individual, familial, and societal distress.

Our data suggests that referring physicians often correctly identify certain positive motor phenomena such as tremor and dyskinesia but frequently miss negative phenomena such as bradykinesia and rigidity. Negative motor findings may not be obvious with informal observation alone and require a formal motor examination that specifically assesses for their presence or absence. In contrast to dyskinesia and tremor, referring clinicians had difficulty identifying the positive motor phenomena of dystonia and chorea. This may indicate that community physicians are more familiar with tardive dyskinesia and tremor because of their frequent association with medication exposure ${ }^{9}$.

Multiple assessment tools have been developed to identify the presence of abnormal movements. Two of these scales, the Extrapyramidal Symptom Rating Scale (ESRS) ${ }^{10}$ and the Abnormal Involuntary Movement Scale (AIMS) ${ }^{11}$ are commonly reported in the psychiatric literature and are suggested for clinical implementation for identification of movement disorders by non-movement disorders specialists ${ }^{12}$. It is possible that use of tools such as the ESRS or AIMS could improve the phenomenology and diagnostic accuracy in identifying abnormal movements. However, these scales require clinicians to accurately assess and describe movements through physical examination. Failure to identify movements accurately could negatively impact the validity of these assessments. Our findings suggest that in addition to the use of such tools, there may be the need for increased focus on the education of community practitioners on the accurate assessment, identification and labeling of abnormal movements.

There were no significant gender differences in our study but the number of referrals increased with patient age. It has been well established that the risk of spontaneous as well as antipsychotic induced abnormal movements in the psychiatric population increases with age ${ }^{13}$. Further, the prevalence of many primary movement disorders (e.g. essential tremor and Parkinson's disease) increases with age ${ }^{14}$. The age of our patient sample is consistent with Lauterbach, et $\mathrm{al}^{2}$.

The mean interval between onset of abnormal movements and referral to the MD clinic was 5.2 years. This mean duration of abnormal movements prior to referral to a movement disorders specialist is consistent with reports from other studies ${ }^{2}$. Delays may be the result under-recognition by either the patient or primary clinician or because of attempts to treat prior to making the referral.

The most common psychiatric diagnoses in our study were: affective disorders (33\%), schizophrenia (23\%), bipolar disorder $(14 \%)$ and schizoaffective disorder $(10 \%)$. These are the patient sub-groups most likely to be exposed to long-term antipsychotics and therefore at risk for a DIMD. Furthermore, abnormal movements are also common in drug naïve psychiatric patients with schizophrenia and affective disorders ${ }^{13,15}$.

In our study, DIMD comprised more than half of the movement disorder diagnoses (51\%) and may represent a significant proportion of those in which the etiology was uncertain. The recommendation by the consulting service was to change or adjust antipsychotics in $17 \%$ of the entire cohort based on evidence that atypical neuroleptics confer less risk of adverse motor outcomes ${ }^{16}$. Further, switching from a typical antipsychotic to quetiapine may result in a significant reduction in pre-existing extrapyramidal symptoms ${ }^{17}$. However, adjusting the psychiatric medications to alleviate motor dysfunction needs to be balanced by the need for effective treatment of psychiatric illness. Emerging evidence suggests that switching antipsychotics may be associated with substantially poorer psychiatric outcomes and greater health care costs ${ }^{18}$.

Our findings are consistent with Lauterbach et al in that we report the presence of a large variety of movement disorders in our sample and abnormal movements were not restricted to just DIMD or tardive disorders ${ }^{2}$. Our data, particularly the discrepancy between drug induced diagnostic labels assigned by community physicians $(82 \%)$ vs. the movement disorders clinic $(51 \%)$ indicates a possible bias among community referral sources towards providing drug-induced phenomenology and diagnostic labels in a psychiatric population.

The present study does have some weaknesses. The participants were intentionally selected from a joint movement disorders and neuropsychiatry clinic which may have biased the sample. Additionally, our comparisons are based on the clinical judgment of a single, although well experienced, movement disorders specialist. As well, this is a retrospective chart review, which has some inherent issues such as difficulty controlling for the accuracy of the records, controlling bias, and confounders. However, we balanced this by reviewing charts of all individuals seen in the clinic.

While the design of our study does not allow us to draw conclusions about the impact of symptom presentation variability on our findings, we think it is unlikely this explains all of the effect seen. In our study, the nature of the lack of concordance was not strictly the failure of identification of an abnormal movement (e.g. failure to identify tics) but also the suspected mislabeling of a movement. For example stereotypies were labeled as tremor. Lastly, while our phenomenology consensus has a substantial samples size, our diagnostic consensus study is based on a small number of cases (approximately $1 / 3$ of the sample of diagnostic terms), which may bias results toward the diagnoses that community referrals were comfortable diagnosing and as such over-inflate the overall inter-rater agreement for diagnosis. 


\section{Conclusions}

The correct diagnosis and appropriate management of movement disorders in patients with psychiatric illness can be challenging. Our study suggests that clinicians would benefit from educational resources to assist them in the identifying and labeling abnormal movements and assigning diagnostic terms that facilitate accurate treatment of these conditions. Access to models where psychiatrists, community practitioners, and neurologists collaborate to optimize the psychiatric health of patients with concomitant movement disorders may facilitate knowledge translation and benefit care. To our knowledge, this study is the first to offer a unique view into the concordance of both phenomenology and diagnostic terminology between community physicians and a movement disorders clinic in a sample of community living individuals with a large variety of psychiatric diagnosis.

\section{Disclosures}

Ms. Angela Roberts-South - Receives PhD funding from Parkinson Society Canada

Dr. Leonardo Cortese - Leonardo Cortese has served on the advisory boards of Eli Lilly, Janssen-Ortho, Lundbeck, Novartis, AstraZeneca, Pfizer, Prestwick, GlaxoSmithKline, and BristolMyers Squibb. He has received grants from Eli Lilly, JanssenOrtho, Astra Zeneca, Pfizer, and Senofi-Aventis. There are no competing interests or conflicts as pertains to this study.

Dr. Mandar Jog - Aging and Mobility Grant Canadian Institutes of Health Research; Research Grant Merz Pharmaceuticals; Research Grant Novartis Canada; Research Grant Teva; Pilot Study Grant Parkinson Society Canada; Research support salary award Academic Medical Organization Southwest Ontario. Dr. Jog serves on scientific advisory boards for Allergan (Chair 2008, 2009); Novartis (participant 2008 2011); Biovail (participant 2009- 2011); Teva (Chair/participant 2008-2011). Travel grants for conference proceedings Teva 20082011 and Novartis 2009-2011. He has served as the Associate Editor for the Canadian Journal of Neurological Sciences. He has received honoraria from Novartis, Allergan, Biovail Pharma, and Teva Neuroscience, Merz pharmaceutical, Boehringer Ingelheim, Prestwick Pharmaceuticals, GlaxoSmithKline.

\section{REFERENCES}

1. Hansen TE, Brown WL, Weigel RM, Casey DE. Underrecognition of tardive dyskinesia and drug-induced parkinsonism by psychiatric residents. Gen Hosp Psychiatry. 1992;14: 340-44.

2. Lauterbach EC, Carter WG, Rathke KM, et al. Tardive dyskinesia-diagnostic issues, subsyndromes, and concurrent movement disorders: a study of state hospital inpatients referred to a movement disorder consultation service. Schizophr Bull. 2001; 27:601-13.

3. Llau ME, Nguyen L, Senard JM, et al. Drug-induced parkinsonian syndromes: a 10-year experience at a regional center of pharmaco-vigilance. Rev Neurol (Paris). 1994;150:757-62.

4. Nguyen N, Pradel V, Micallef J, et al. Drug-induced parkinson syndromes. Therapie. 2004;59(1):105-12.

5. Stemler SE. A comparison of consensus, consistency, and measurement approaches to estimating interrater reliability. Practical Assessment, Research \& Evaluation. 2004;9(4).

6. Albanese A, Barnes MP, Bhatia KP, et al. A systematic review on the diagnosis and treatment of primary (idiopathic) dystonia and dystonia plus syndromes: report of an EFNS/MDS-ES Task Force. Eur J Neurol. 2006;13:433-44.

7. Bergen JA, Griffiths DA, Rey JM, Beumont PJ. Tardive dyskinesia: fluctuating patient or fluctuating rater. Br J Psychiatry. 1984; 144:498-502.

8. Chong SA, Tay JA, Subramaniam M, et al. Mortality rates among patients with schizophrenia and tardive dyskinesia. J Clin Psychopharmacology. 2009;29:5-8.

9. Tolosa E, Alom J, Marti MJ. Drug-Induced Dyskinesias. In: Jankovic J, Tolosa E, editors. Parkinson's Disease and Movement Disorders 2nd ed. Baltimore: Williams \& Wilkins, 1993. p. 375-98.

10. Chouinard G, Margolese HC. Manual for the Extrapyramidal Symptom Rating Scale (ESRS). Schizophr Res. 2005;76(2-3): 247-65.

11. Guy W. Abnormal Involuntary Movement Scale (AIMS). ECDEU Assessment Manual for Psychopharmacology - Revised 1976. US Department of Health, Education, and Welfare;

12. Gharabawi M, Bossie C, Laser R, et al. Abnormal Involuntary Movement Scale (AIMS) and Extrapyramidal Symptom Rating Scale (ESRS): cross scale comparison in assessing tardive dyskinesia. Schizophr Res. 2005;77(2-3):119-28.

13. Owens DG, Johnstone EC, Frith CD. Spontaneous involuntary disorders of movement: their prevalence, severity, and distribution in chronic schizophrenics with and without treatment with neuroleptics. Arch Gen Psychiatry. 1982;39: 452-61.

14. Wenning GK, Kiechl S, Seppi K, et al. Prevalence of movement disorders in men and women aged 50-89 years (Bruneck Study cohort): a population-based study. Lancet Neurol. 2005;4: 815-20.

15. Rogers D. The motor disorders of severe psychiatric illness: a conflict of paradigms. Br J Psychiatry. 1985;147:221-32.

16. Tarsy D, Baldessarini RJ. Epidemiology of tardive dyskinesia: is risk declining with modern antipsychotics? Mov Dis. 2006;21: 589-98.

17. Cortese L, Caligiuri MP, Williams R, et al. Reduction in neuroleptic-induced movement disorders after a switch to quetiapine in patients with schizophrenia. J Clin Psychopharmacol. 2008;28:69-73.

18. Faries DE, Ascher-Svanum H, Nyhuis AW, Kinon BJ. Clinical and economic ramifications of switching antipsychotics in the treatment of schizophrenia. BMC Psych. 2009;9:54. 